\title{
The Canadian Institutes of Health Research response to antimicrobial resistance
}

\author{
Pagé $\mathrm{EL}^{1}$, Desnoyers $\mathrm{S}^{1}$, Létourneau $\mathrm{IJ}^{1}$, Keown $\mathrm{K}^{2}$, Jackson $\mathrm{A}^{2}$, Ouellette $\mathrm{M}^{1 \text { * }}$ \\ ${ }^{1}$ Canadian Institutes of Health Research, Institute of Infection and Immunity, Québec, QC \\ ${ }^{2}$ Canadian Institutes of Health Research, Ottawa, ON \\ *Correspondence: marc.ouellette@crchul.ulaval.ca
}

\begin{abstract}
Antimicrobial resistance (AMR) has been a research priority for the Canadian Institutes of Health Research (CIHR), Institute of Infection and Immunity (III) since its inception, and a number of strategic research initiatives have been launched to address this global health problem by promoting and supporting research related to mechanisms and processes that impact the emergence and spread of resistance among individuals and within the environment. Here we will present research initiatives on AMR led by CIHR-III, which include national programs as well as international partnerships with the United Kingdom and the European Union, in addition to interesting outcomes of these initiatives.
\end{abstract}

\section{Introduction}

The Canadian Institutes of Health Research (CIHR) is the Government of Canada's health research investment agency. The Institute of Infection and Immunity (III) is one of the thirteen "virtual" institutes of $\mathrm{CIHR}$ which supports research and builds research capacity in the areas of infectious diseases and immunity in an integrated approach based on four broad themes (bio-medical research; clinical research; research on health systems services; and research on social, cultural, environmental and population health). Based on its 2013-2018 Strategic Plan (1), CIHR-III has developed a number of strategic research initiatives to stimulate targeted areas, including antimicrobial resistance (AMR).

\section{CIHR investments in AMR research and the Federal Action Plan on AMR}

AMR is a growing public health threat that involves interplay between multiple sectors. The loss of effective antimicrobials is reducing the ability to prevent and treat infectious diseases, while also impacting our health care system, global trade, and the agriculture, environment, and health sectors. The World Health Organization has now included AMR as one of the most pressing public health issues. In October 2014, the Government of Canada released Antimicrobial Resistance and Use in Canada: A Federal Framework for Action (2) to serve as a starting point for cohesive engagement and mobilization of all who are accountable for action on AMR and antimicrobial use. Efforts are being focused on three priority areas: Surveillance, Stewardship and Innovation. Through its open and strategic programs, CIHR invested \$93.8 million in AMR research between 2009-2010 and 2013-2014, including over \$15 million in 2013-2014 alone. Most of the funding has been geared toward innovation; moving forward these programs can help to better identify strategies/approaches for stewardship or surveillance.

\section{CIHR-III's strategic research initiatives on AMR}

\section{Antimicrobial resistance, health system implications and health outcomes}

During consultation with the research community for our CIHR-III 2013-2018 Strategic Plan, AMR was clearly identified as a critical strategic research priority. This represents a continuum for CIHR-III, as the Institute has invested in AMR research since its inception. Earlier investments included an Antimicrobial Resistance, Health System Implications and Health Outcomes request for applications resulting in two projects funded. The first funded project was on Antimicrobial Use and Resistance in Seniors and 
studied methicillin-resistant Staphylococcus aureus (MRSA) incidence in elderly hospitalized patients. The team showed that the epidemiology and clinical features of those patients (being more likely to be colonized by MRSA) are different from younger patients (3). The second project, entitled Community-Acquired Antimicrobial Resistant Bacteria in Northern Canadian Communities, led to the creation of the Northern Antibiotic Resistance Partnership (NARP), a team composed of community members, health care professionals, educators and research scientists studying resistant bacteria in northern communities (4). The group has established a surveillance program to monitor bacterial infections and antibiotic use, has developed educational tools for health care providers and the general population, and has produced a community-associated case control study to determine the risk factors associated with community-acquired MRSA (CA-MRSA) infections in northern regions of Saskatchewan.

\section{Safe Food and Water Research Initiative}

The Safe Food and Water Research Initiative, which was sparked in part by the Walkerton tragedy, brought together governmental and private sector organizations in order to develop a national research strategy to prevent and respond to diseases caused by foodborne and/or waterborne pathogens or their toxins. The two requests for applications launched resulted in the funding of four projects related to AMR-a \$2.8 million investment in total. Outcomes of this investment include the establishment of a baseline of resistance profiles and the mechanisms of resistance observed in Campylobacter jejuni in poultry from Alberta (5), and the characterization of cefotoxin-resistant Escherichia coli strains from Canadian water sources, as well as prevention measures to avoid bacterial contamination of water streams $(6,7)$.

\section{Novel Alternatives to Antibiotics Initiative}

The Novel Alternatives to Antibiotics Initiative was designed to enhance the existing funding available through the CIHR open competitions by attracting applications focused on novel approaches to antibiotic resistance in which Canada had little or no research capacity. Three priority themes were determined: immune systems; phage therapy (or the use of viruses to infect and kill pathogenic bacteria); and physical systems and biomaterials. This investment was the product of the collaboration of several public and private sector partners and the funded projects resulted in several interesting outcomes, including promising results using a non-invasive phage delivery technique through aerosol to treat respiratory tract infection (8) and the identification of new antibiotics against MRSA. Several patents have been filed and intellectual property has been transferred to a Canadian spin-off company following the latter project (9).

\section{The Canada/U.K. partnership on antimicrobial resistance}

Since 2007, Canada has partnered with the Medical Research Council in the United Kingdom, resulting in several workshops and joint competitions. Following an initial workshop and catalyst funding, in September 2010, a team grant involving a partnership between Canada and the U.K. on antibiotic resistance was launched that built on existing collaborations between the two countries and provided four years of support. The first team funded dealt with bacterial cell wall synthesis and is in the process of testing several potential inhibitors with the aim of identifying leads for novel antibiotics (10-12). The second team studied bacterial resistance to $\beta$-lactam antibiotics which led to multiple outcomes, including the design, synthesis and testing of new candidate inhibitors of metallo- $\beta$-lactamases, and the characterization of inhibition of key metallo $\beta$-lactamases targets by known compounds $(13,14)$. The Canada/U.K. partnership investment was close to $\$ 4$ million and allowed the funded teams to secure additional funding and partnerships as well as building capacity in both areas. A second workshop, on Translational Strategies to Combat Antibiotic Resistance, followed in 2013 and provided recommendations for increasing awareness, supporting public and private sector partnerships, increasing financial investments and supporting collaborative approaches (15).

\section{Joint Programming Initiative on Antimicrobial Resistance}

Canada, through CIHR, is a major funder of the Joint Programming Initiative on Antimicrobial Resistance (JPIAMR), an international network of 20 countries providing a collaborative platform to take AMR from awareness to action by supporting research and facilitating its translation to industry and policy. The aim of this initiative is to develop integrated approaches to pursue unique world-class research on AMR. Through its Strategic Research Agenda, JPIAMR enhances multidisciplinary collaboration and ensures that knowledge gaps are quickly identified and filled. Actions within six priority topics (therapeutics, diagnostics, 
surveillance, transmission, environment, and interventions) will be translated into new prevention and intervention strategies to improve public health and deliver economic and societal benefits. Launched in 2014, the transnational call InnovaResistance: Innovative Approaches to Address Antibacterial Resistance resulted in the funding of seven consortia, six of which include Canadian researchers (16). The primary aim of this joint call is to combine the resources, infrastructures and research strengths of multiple countries in order to overcome antibiotic resistance. The call focused on the re-evaluation of existing antimicrobial compounds (alone or in combination with other drugs, immune-modulators or antibacterial approaches), identification of new bacterial targets and/or therapeutic compounds, discovery of novel therapies to overcome known antimicrobial resistance mechanisms and restore susceptibility to conventional antibiotics, or drug combinations and strategies to inhibit or reduce the acquisition of resistance. The Canadian nominated projects were in line with several of those priorities.

Recently JPIAMR announced another transnational call to be launched in early 2016 in which 20 funding organizations throughout the world will take part, including Canada. The topic of that call will promote research to "unravel the dynamics of transmission and selection of antimicrobial resistance (AMR) at genetic, bacterial, animal, human, societal, and environmental levels, in order to design and evaluate preventive and intervening measures for controlling resistance" (17).

JPIAMR is dedicated to the One Health approach where interdisciplinary collaborations are created to tackle health care issues. Hence, investigation of the mechanisms leading to the spread of resistance in and between different reservoirs, including animals, the environment and people, will contribute to the design of preventive measures to address the public health threat of AMR.

\section{Other CIHR strategic investments}

Related to the One Health approach, CIHR has developed the Environments and Health Signature Initiative which integrates a nexus approach (three nexus areas) through the study of cumulative environmental exposures, and their interconnections, intersections and impacts on health and disease across the life course (18). The JPIAMR transnational call to come in 2016 aligns with the topics of the Environments and Health Signature Initiative. Both will support research to examine how intersectoral strategies and approaches contribute to improvements in population health and health equity.

\section{Conclusion}

Antimicrobial resistance is a serious problem that causes the hospitalization of more than 250,000 Canadians every year, and more than 18,000 hospitalized patients acquire drug-resistant infections (19). For instance, deaths directly related to Clostridium difficile alone have increased five-fold in the past decade (20). It is also a severe economic drain. Initial research has shown that if no action is taken, a continued rise of resistance would lead, in 2050, to 10 million deaths and a cost of \$100 trillion USD worldwide (21). CIHR-III is committed to improving the lives of Canadians and the global community by supporting innovative research in AMR. In the past five years, CIHR and III have invested more than \$93.8 million in combatting AMR. AMR research will remain a focus as the threat continues to expand. CIHR and III play a proactive role in the Federal Action Plan on AMR and will continue to promote innovation by supporting national and international collaborations and multi-theme initiatives in order to allow leveraging of investments. Future strategic research investments will deal with both the development of novel molecules but also with strategies to protect the current antibiotics available through better diagnostics and stewardship. There is currently momentum in the research field as well as in the policy arena to find innovative solutions for tackling the vexing problem of AMR, which has been coined by the 2013 World Economic Forum as the greatest risk of hubris to human health. 


\section{Acknowledgements}

The authors wish to thank the previous administration team of CIHR-III, under the direction of Dr. Bhagirath Singh, for its hard work on AMR programs. Special thanks to the dedicated AMR team working at CIHR.

\section{Conflict of interest}

None

\section{References}

(1) Canadian Institutes of Health Research. Institutes-Infection and Immunity. III Strategic Plan 2013-18. 2013 Dec. http://www.cihr-irsc.gc.ca/e/46554.html

(2) Government of Canada. Antimicrobial Resistance and Use in Canada: A Federal Framework for Action. Ottawa, ON: Public Healty Agency of Canada; 2014 Oct. http://healthycanadians.gc.ca/alt/pdf/drugs-products-medicaments-produits/buying-using-achatutilisation/antibiotic-resistance-antibiotique/antimicrobial-framework-cadre-antimicrobiens-eng.pdf

(3) Simor AE, Ofner-Agostini M, Paton S, McGeer A, Loeb M, Bryce E, et al. Clinical and epidemiologic features of methicillin-resistant Staphylococcus aureus in elderly hospitalized patients. Infect Control Hosp Epidemiol. 2005 Oct;26(10):838-41.

(4) Northern Antibiotic Resistance Partnership (NARP). http://www.narp.ca/

(5) Kos VN, Keelan M, Taylor DE. Antimicrobial susceptibilities of Campylobacter jejuni isolates from poultry from Alberta, Canada. Antimicrob Agents Chemother. 2006 Feb;50(2):778-80.

(6) Mataseje LF, Neumann N, Crago B, Baudry P, Zhanel GG, Louie M, et al. Characterization of cefoxitin-resistant Escherichia coli isolates from recreational beaches and private drinking water in Canada between 2004 and 2006. Antimicrob Agents Chemother. 2009 Jul;53(7):3126-30. doi: 10.1128/AAC.01353-08. Epub 2009 Apr 27.

(7) Zhu Z, Broersma K, Mazumder A. Model assessment of cattle and climate impacts on stream fecal coliform pollution in the Salmon River watershed, British Columbia, Canada. Water Air Soil Pollut. 2011;215:155-76. doi: 10.1007/s11270-010-0467-0.

(8) Semler DD, Goudie AD, Finlay WH, Dennis JJ. Aerosol phage therapy efficacy in Burkholderia cepacia complex respiratory infections. Antimicrob Agents Chemother. 2014 Jul;58(7):4005-13. doi: 10.1128/AAC.02388-13. Epub 2014 May 5.

(9) DeNovaMed. http://www.denovamed.com

(10) Farha MA, Czarny TL, Myers CL, Worrall LJ, French S, Conrady DG, et al. Antagonism screen for inhibitors of bacterial cell wall biogenesis uncovers an inhibitor of undecaprenyl diphosphate synthase. Proc Natl Acad Sci USA. 2015 Sep 1;112(35):11048-53. doi: 10.1073/pnas.1511751112. Epub 2015 Aug 17.

(11) Kouidmi I, Levesque RC, Paradis-Bleau C. The biology of Mur ligases as an antibacterial target. Mol Microbiol. 2014 Oct;94(2):242-53. doi: 10.1111/mmi.12758. Epub 2014 Sep 5.

(12) Czarny TL, Perri AL, French S, Brown ED. Discovery of novel cell wall-active compounds using $\mathrm{P}$ ywaC, a sensitive reporter of cell wall stress, in the model gram-positive bacterium Bacillus subtilis. Antimicrob Agents Chemother. 2014 Jun;58(6):3261-9. doi: 10.1128/AAC.02352-14. Epub 2014 Mar 31.

(13) Ghavami A, Labbé G, Brem J, Goodfellow VJ, Marrone L, Tanner CA, et al. Assay for drug discovery: Synthesis and testing of nitrocefin analogues for use as $\beta$-lactamase substrates. Anal Biochem. 2015 Oct 1;486:75-7. doi: 10.1016/j.ab.2015.06.032. Epub 2015 Jul 2.

(14) Rotondo CM, Marrone L, Goodfellow VJ, Ghavami A, Labbé G, Spencer J, et al. Arginine-containing peptides as potent inhibitors of VIM-2 metallo- $\beta$-lactamase. Biochim Biophys Acta. 2015 Nov;1850(11):2228-38. doi: 10.1016/j.bbagen.2015.07.012. Epub 2015 Aug 1.

(15) Canadian Institutes of Health Research (CIHR). Translational Strategies to Combat Antibiotic Resistance: A Call to Action-Workshop Report. A Canada/UK Collaboration between: Canadian Institutes of Health Research, Institute of Infection and Immunity, UK Health Protection Agency, Canadian High Commission. Canada House, London, UK. 2013 Feb 6-7. Ottawa, ON: CIHR; 2014.

http://www.cihr-irsc.gc.ca/e/48215.html

(16) Joint Programming Initiative on Antimicrobial Resistance (JPIAMR). Projects. Results from the first JPIAMR transnational call InnovaResistance: Innovative approaches to address antibacterial resistance. 2014. http://www.jpiamr.eu/wp-content/uploads/2014/12/Results-from-the-first-transnational-callInnovaResistance1.pdf

(17) Joint Programming Initiative on Antimicrobial Resistance (JPIAMR). News. Save the date: Transmission dynamics call for proposals to open in January 2016. 2015 Jul 23.

http://www.jpiamr.eu/save-the-date-transmission-dynamics-call-for-proposals-to-open-in-january-2016/ 
(18) Canadian Institutes of Health Research. Initiatives. Environments and Health: Overview. 2015 Feb 19. http://www.cihr-irsc.gc.ca/e/48465.html

(19) Zoutman DE, Ford BD, Bryce E, Gourdeau M, Hébert G, Henderson E, et al. The state of infection surveillance and control in Canadian acute care hospitals. Am J Infect Control. 2003 Aug;31(5):266-72; discussion 272-3.

(20) Gravel D, Miller M, Simor A, Taylor G, Gardam M, McGeer A, et al. Health care-associated Clostridium difficile infection in adults admitted to acute care hospitals in Canada: A Canadian Nosocomial Infection Surveillance Program study. Clin Infect Dis. 2009 Mar 1;48(5):568-76.

(21) O'Neill J. Antimicrobial Resistance: Tackling a crisis for the health and wealth of nations. The Review on Antimicrobial Resistance. 2014 Dec. http://amr-review.org/Publications 